\title{
Erratum
}

\section{Erratum to "Joint Delay Doppler Probability Density Functions for Air-to-Air Channels"}

\author{
Michael Walter, Dmitriy Shutin, and Uwe-Carsten Fiebig \\ Institute of Communications and Navigation, German Aerospace Center (DLR), Oberpfaffenhofen, 82234 Wessling, Germany \\ Correspondence should be addressed to Michael Walter; m.walter@dlr.de
}

Received 6 August 2014; Accepted 8 September 2014; Published 28 October 2014

Copyright (C) 2014 Michael Walter et al. This is an open access article distributed under the Creative Commons Attribution License, which permits unrestricted use, distribution, and reproduction in any medium, provided the original work is properly cited.

In the article titled "Joint delay Doppler probability density functions for air-to-air channels," International Journal of Antennas and Propagation, Volume 2014 (2014), Article ID 814218, 11 pages, http://dx.doi.org/10.1155/2014/814218, errors occurred in (14), (19), and (20).

(I) Equation (14). The semimajor axis $a_{\mathrm{ell}}$ and the semiminor axis $b_{\text {ell }}$ in (14) have to be calculated as

$$
\begin{gathered}
a_{\mathrm{ell}}=\sqrt{\left(\frac{p}{a}\right)^{2}-\frac{d}{a}} \\
b_{\mathrm{ell}}=\sqrt{\frac{p^{2}}{a b}-\frac{d}{b}} .
\end{gathered}
$$

The offset from the origin $\left[x_{c}, y_{c}\right]$ is correct.

(II) Equation (19). It should read as follows:

$$
p(\phi \mid \tau)=\frac{\sqrt{1-\epsilon_{\tau}^{2} \cos ^{2} \phi}}{4 E\left(\epsilon_{\tau}\right)} .
$$

(III) Equation (20). It should read as follows:

$$
\begin{aligned}
& p(\phi \mid \tau ; \kappa, \mu) \\
& =\frac{\sqrt{1-\epsilon_{\tau}^{2} \cos ^{2} \phi}}{4 E\left(\epsilon_{\tau}\right)} \\
& \quad \times \frac{\exp \left\{\kappa \cos \left(\left(\pi / 2 E\left(\epsilon_{\tau}\right)\right)\left(\int_{0}^{\phi} \sqrt{1-\epsilon_{\tau}^{2} \cos ^{2} \zeta} \mathrm{d} \zeta-\mu\right)\right)\right\}}{I_{0}(\kappa)},
\end{aligned}
$$

with $\kappa$ being the concentration parameter of the distribution and $\mu$ being the centrality parameter of the distribution, that is, the place on the ellipse, where the dominant scatterers come from. The centrality parameter is given by $\mu=$ $\int_{0}^{\bar{\phi}} \sqrt{1-\epsilon_{\tau}^{2} \cos ^{2} \zeta} \mathrm{d} \zeta$ and $I_{0}(\kappa)$ is the modified zeroth order Bessel function of the first kind. 

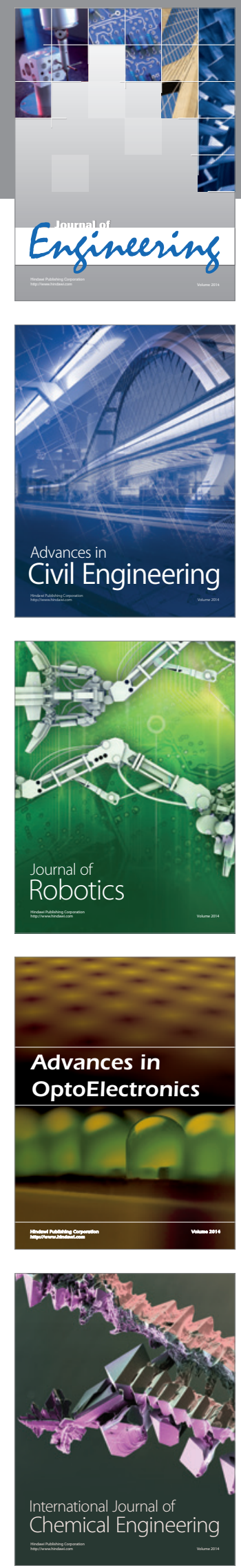

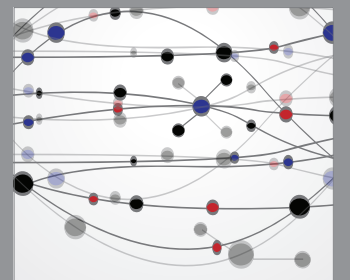

The Scientific World Journal
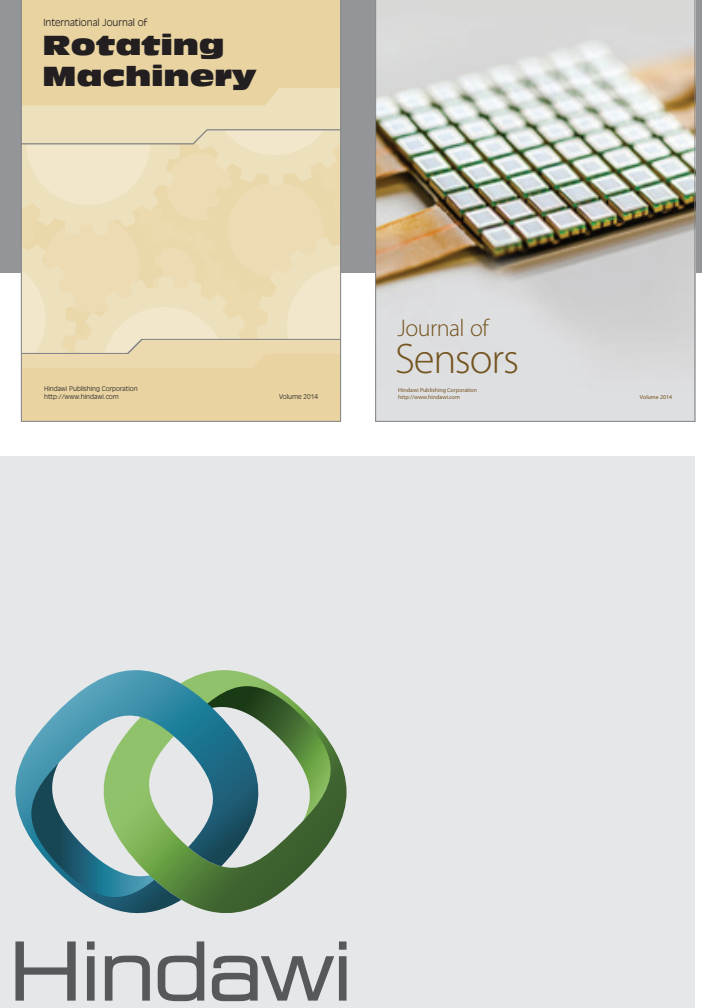

Submit your manuscripts at http://www.hindawi.com
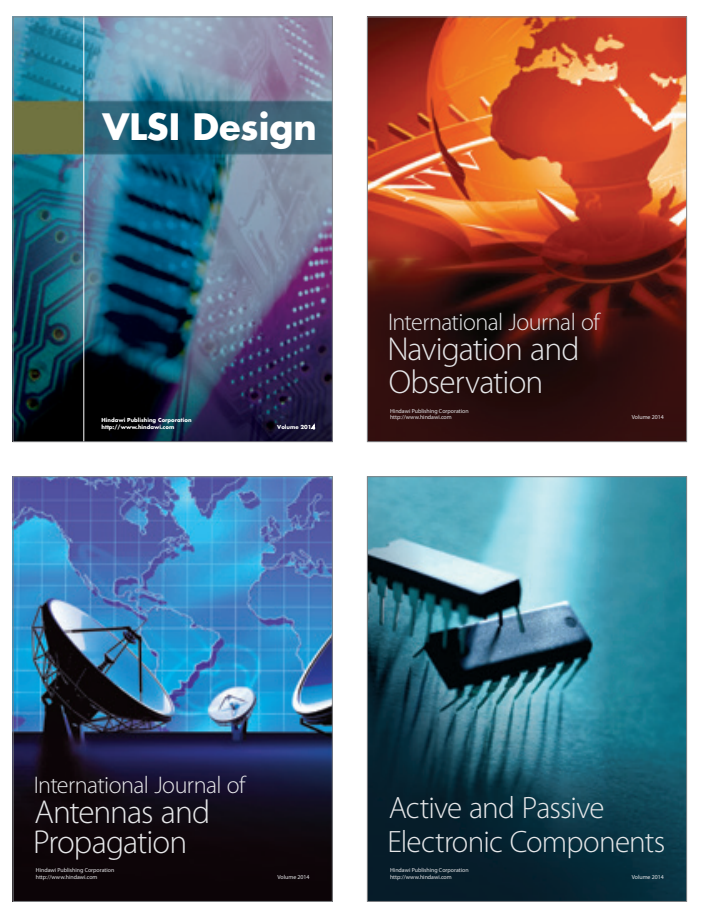
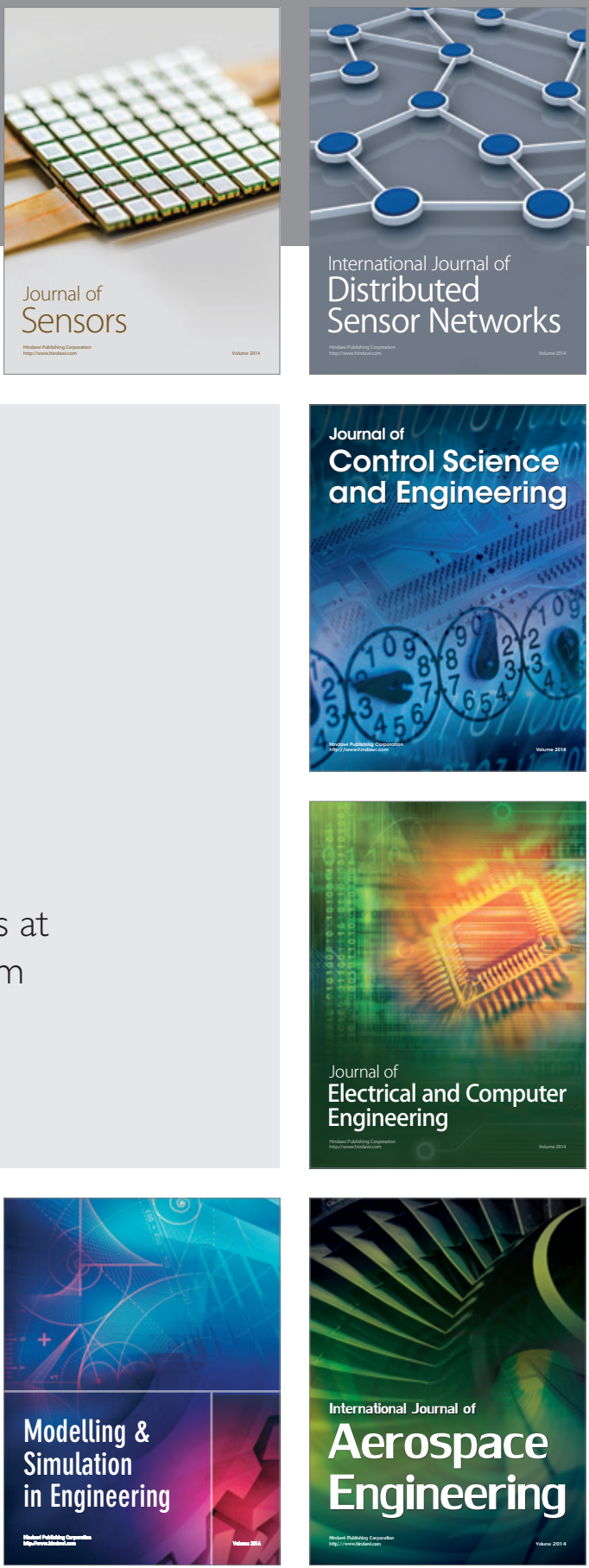

Journal of

Control Science

and Engineering
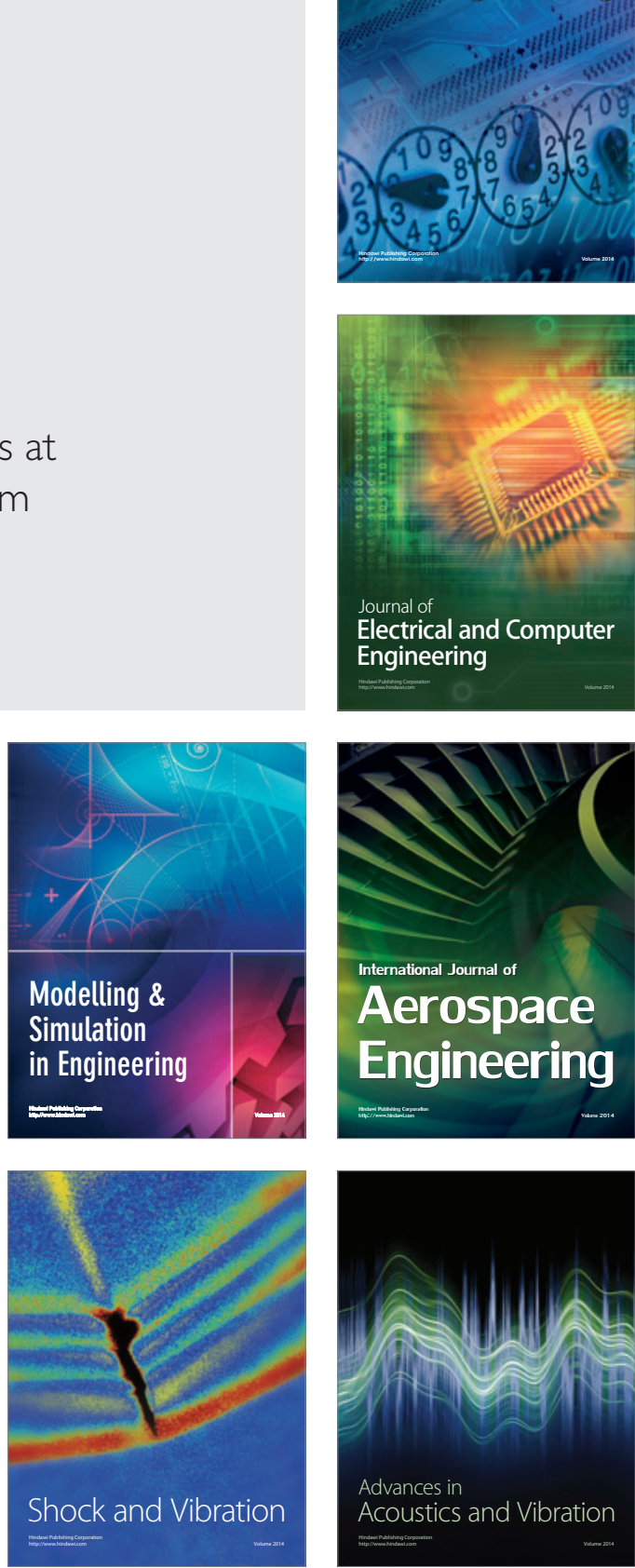